\title{
Hotter Is Better and Broader: Thermal Sensitivity of Fitness in a Population of Bacteriophages
}

\author{
Jennifer L. Knies, ${ }^{1,2, *}$ Joel G. Kingsolver, ${ }^{1}$ and Christina L. Burch ${ }^{1}$ \\ 1. Department of Biology, University of North Carolina, Chapel Hill, North Carolina 27599; 2. Curriculum in Genetics and Molecular \\ Biology, University of North Carolina, Chapel Hill, North Carolina 27599 \\ Submitted January 4, 2008; Accepted January 17, 2008; Electronically published February 20, 2009
}

\begin{abstract}
Hotter is better is a hypothesis of thermal adaptation that posits that the rate-depressing effects of low temperature on biochemical reactions cannot be overcome by physiological plasticity or genetic adaptation. If so, then genotypes or populations adapted to warmer temperatures will have higher maximum growth rates than those adapted to low temperatures. Here we test hotter is better by measuring thermal reaction norms for intrinsic rate of population growth among an intraspecific collection of bacteriophages recently isolated from nature. Consistent with hotter is better, we find that phage genotypes with higher optimal temperatures have higher maximum growth rates. Unexpectedly, we also found that hotter is broader, meaning that the phages with the highest optimal temperatures also have the greatest temperature ranges. We found that the temperature sensitivity of fitness for phages is similar to that for insects.
\end{abstract}

Keywords: hotter is better, adaptation, temperature, exponential temperature dependence.

\section{Introduction}

A central aim of evolutionary biology is to identify patterns of adaptation and use this knowledge to predict evolutionary responses to environmental variation. Adaptation to temperature is an important and useful model for investigating evolutionary responses (Precht et al. 1973; Johnston and Bennett 1996) because temperature affects nearly all biological rate processes (Bowler and Cossins 1987; Gillooly et al. 2001), and our knowledge of the thermodynamic effects of temperature can be used to predict the existence and nature of constraints on temperature adaptation. For example, low temperatures are known to depress reaction rates by reducing the collision rate of reactants and the fraction of molecules that have sufficient energy to be kinetically active (Hochachka and Somero

\footnotetext{
* Corresponding author. Present address: Department of Ecology and Evolutionary Biology, Brown University, Providence, Rhode Island 02912; e-mail: jennifer_knies@brown.edu.

Am. Nat. 2009. Vol. 173, pp. 419-430. () 2009 by The University of Chicago. 0003-0147/2009/17304-50143\$15.00. All rights reserved.

DOI: $10.1086 / 597224$
}

2002). It is not known whether evolutionary adaptation at low temperatures can overcome this depression in reaction rates.

In this article, we test two aspects of a hypothesis of thermal adaptation that arises from this potential constraint on adaptation to cold temperatures (Hamilton 1973; Savage et al. 2004). The original hypothesis, hotter is better, stems from the assumption that adaptation is unable to overcome the rate-depressing effects of low temperature. As a result, organisms adapted to lower temperatures are predicted to have lower maximum performances (e.g., sprinting speeds, fitness, and growth rates) than those adapted to higher temperatures (Bennett 1987). Considered in terms of continuous reaction normscurves that relate a continuous trait (e.g., performance) to a continuous environmental variable (e.g., temperature)-hotter is better predicts a positive correlation between an organism's optimal temperature and its maximum performance (i.e., performance at the optimal temperature; Hamilton 1973; Huey and Kingsolver 1989; Frazier et al. 2006; fig. 1A). In contrast, if organisms living at low temperatures can compensate for rate-depressing effects (Huey and Kingsolver 1989), then organisms inhabiting low temperatures should achieve the same maximum performances as those living at high temperatures (Frazier et al. 2006; fig. 1B).

Experiments that address the hotter is better hypothesis have produced mixed results. The strongest support for hotter is better comes from comparative studies between species (or higher taxa; Eppley 1972; Frazier et al. 2006). For example, comparative studies on lizard sprinting speed (Bauwens et al. 1995), pine tree growth (Rehfeld et al. 2002), scallop metabolism (Heilmayer et al. 2004), and insect rates of population growth (Frazier et al. 2006) all showed the hotter is better pattern that species with higher optimal temperatures had higher maximal performances. However, because these comparisons are among species separated by long evolutionary time periods, it is difficult to determine whether the observed pattern reflects a limitation on adaptation or whether differences among species 
resulted from evolutionary constraints that are correlated with, but not caused by, temperature. If the hotter is better pattern that characterizes comparisons between species reflects limitations on adaptation, then the same pattern should characterize comparisons between genotypes within species. Indeed, such intraspecific comparisons should more directly reflect limitations on the adaptation of reaction norms. To date, intraspecific studies (e.g., of invertebrate locomotor speed [Herron 1996] and caterpillar growth rate [Izem and Kingsolver 2005]) have not shown the hotter is better pattern, making it unclear whether the thermodynamic effects of low temperature impose a substantial constraint on temperature adaptation.

Another important feature of an organism's thermal biology is its temperature range: the range of temperatures over which an organism can achieve a fitness greater than its replacement rate (Levins 1968; Gilchrist 1995; Portner 2004). A common assumption is that there are trade-offs between thermal specialists and thermal generalists, such that maximal performance or fitness is inversely related to temperature range. As a result, selection for an increased temperature range is expected to result in a reduction in maximal performance (Levins 1968; Huey and Slatkin 1976). Conversely, selection for maximal performance is expected to reduce an organism's temperature range. If such trade-offs exist, then hotter is better can be achieved only through a narrowing of reaction norms. Similarly, if there is selection for an increased temperature range in cold-adapted species as a result of greater thermal variability in cooler environments, generalist-specialist tradeoffs would result in greater temperature range, lower optimal temperature, and lower maximal performance in cold-adapted species (Frazier et al. 2006). These effects would result in a negative correlation between an organism's optimal temperature and temperature range (fig. $1 C)$, causing "hotter is narrower."

Despite the widespread assumption of generalistspecialist trade-offs in evolutionary models of thermal adaptation (Lynch and Gabriel 1987; Gilchrist 1995), empirical support for such trade-offs is weak (Huey and Hertz 1984; Angilletta et al. 2002; Yamahira et al. 2007). If generalist-specialist trade-offs do not constrain adaptation to temperature, then hotter is better could generate a higher and broader reaction norm, increasing the temperature range (fig. $1 D$ ).

Here, we provide a new test of both hotter is better and hotter is narrower by comparing thermal reaction norms between genotypes within an intraspecific sample of G4like bacteriophages collected from nature. In this model system, it is possible to measure thermal reaction norms with a high level of precision. A previous experiment examined the thermal reaction norms of G4 phages adapted to high temperature in the lab (Knies et al. 2006) and found results consistent with hotter is better but had too few phage genotypes (four) to be conclusive. As in the prior experiment, we ensured accurate estimates of optimal temperature $\left(T_{\mathrm{opt}}\right)$, maximum intrinsic growth rate $\left(r_{\max }\right)$, and temperature range by measuring the intrinsic growth rates (our fitness measure) of 15 natural isolates of G4-like bacteriophages at numerous temperatures across a wide temperature range. To test hotter is better and hotter is narrower, we computed the correlation between the phage's $T_{\text {opt }}$ and $r_{\max }\left(r\right.$ at $\left.T_{\text {opt }}\right)$ and also between the phage's $T_{\mathrm{opt}}$ and temperature range (range of temperatures for which $r>0$ ). We also calculated the thermal sensitivity of $\ln r_{\max }$ for phages, or the rate at which $\ln r_{\max }$ increases with $T_{\text {opt }}$ (Gillooly et al. 2001), and compare our results with those for insects (Frazier et al. 2006).

\section{Methods}

\section{Strains and Culture Conditions}

G4 is a single-stranded DNA phage whose standard lab host is the bacterium Escherichia coli strain C (Fiddes et al. 1978). The 15 phages used in this study are described by Rokyta et al. (2006) and were obtained from H. Wichman (University of Idaho, Moscow). These phages were isolated from samples collected from barns and sewage treatment plants in Washington (WA), Idaho (ID), and North Carolina (NC) in 2001 and 2002. Samples were plated directly onto a lawn of $E$. coli strain $\mathrm{C}$ and incubated at $37^{\circ} \mathrm{C}$ until visible plaques formed. The genomes of 42 of the resulting phage isolates were sequenced and used to reconstruct the phylogenetic relationships between them. These phages formed three distinct monophyletic groups, with one of them containing G4, another containing the phage $\phi \mathrm{X} 174$, and the third containing the phage $\alpha 3$. This G4-like clade contained 14 phages that were closely related to $\mathrm{G} 4$, with a maximum pairwise difference of $9 \%$, and three additional phages that were substantially less related. Here we examine only G4 and the closely related members of the G4-like clade because they form a single species according to the phylogenetic species concept of Palys et al. (1997; sequence clusters with ratios of average pairwise between-group to within-group distances of $>2$ are indicative of separate species). Following the naming given by Rokyta et al. (2006), the natural isolates used in this article are as follows: ID12, NC19, NC13, NC6, WA5, NC2, ID52, ID11, NC10, WA2, WA3, ID8, ID41, WA6, and G4, where the two-letter prefix (excepting G4) indicates the state in which the phage was found.

Culture conditions were as described by Holder and Bull (2001) and Knies et al. (2006). Briefly, phages were grown on E. coli strain C, the standard laboratory host of G4, in 

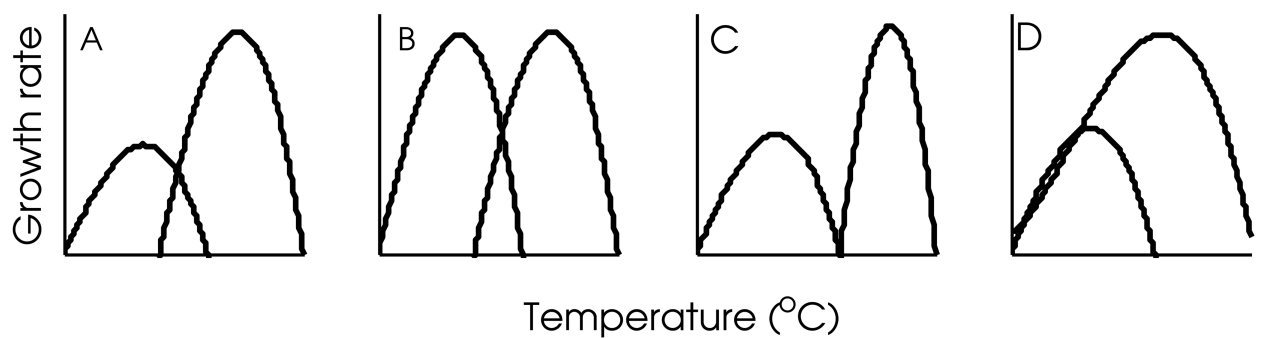

Temperature $(e \mathrm{C})$

Figure 1: Competing hypotheses predicting how maximum growth rate (fitness, $r$ ) and temperature range evolve in response to temperature adaptation. $A$, Hotter is better predicts that adaptation to higher temperatures results in increased maximum growth rate. $B$, If thermodynamic constraints do not constrain adaptation to temperature, then adaptation to high and low temperatures is predicted to result in equal maximal growth rates. $C$, In combination with hotter is better, generalist-specialist trade-offs predict that populations adapted to higher temperatures will have a narrow temperature range. $D$, If generalist-specialist trade-offs or other environmental effects do not constrain temperature adaptation, then populations adapted to the higher temperatures are predicted to have the wider temperature ranges.

Luria broth (LB; $5 \mathrm{~g}$ yeast extract, $10 \mathrm{~g}$ Bacto tryptone, 5 g salt/1 L) supplemented to $2 \mathrm{mM} \mathrm{CaCl}_{2}$, and on LB plates ( $15 \%$ agar). LB top agar $(0.7 \%$ agar) was also supplemented to $2 \mathrm{mM} \mathrm{CaCl}_{2}$. Phage were stored for short term $\left(<2\right.$ weeks) at $4^{\circ} \mathrm{C}$ in this growth media and for long term at $-20^{\circ} \mathrm{C}$ in $\mathrm{LB}$ broth supplemented to $2 \mathrm{mM} \mathrm{CaCl}_{2}$ and $40 \%$ glycerol.

\section{Growth Rate (Fitness) Assays}

Growth rate assays were as described by Knies et al. (2006). Escherichia coli strain C cultures were grown overnight at the experimental temperature, and on the following day, $60 \mu \mathrm{L}$ of this stationary phase E. coli strain C culture was used to inoculate $2 \mathrm{~mL}$ of $\mathrm{LB}$ (containing $2 \mathrm{mM} \mathrm{CaCl}_{2}$ ). This fresh culture was incubated at the experimental temperature until the culture reached an optical density $\left(\mathrm{OD}_{600}\right)$ of $0.6 \pm 0.06$, which corresponds to $2-3 \times 10^{8}$ cells $/ \mathrm{mL}$. Approximately $10^{3}-10^{4}$ phages were added to this exponentially growing culture, and the mixture was grown for $45 \mathrm{~min}$, at which point the culture was treated with $100 \mu \mathrm{L}$ chloroform. Chloroform lyses the bacteria, and there are typically very few mature phage inside a cell prelysis except for immediately before lysis, when the number of mature phage approaches the burst size (J. L. Knies and C. L. Burch, unpublished data). Therefore, plating from this mixture of chloroformed cells gives a count of mature phages, and this count is dominated by free phage plus some small number of mature phage still inside the cells.

Phage numbers at the start $\left(N_{0}\right)$ and end $\left(N_{45}\right)$ of the assay were determined by plating and incubating the plates until well-formed plaques were present. Plates were incubated at $33^{\circ} \mathrm{C}$ for $2.5-4 \mathrm{~h}$ if assays were performed in the morning or at $25^{\circ} \mathrm{C}$ for $16-24 \mathrm{~h}$ if assays were performed in the afternoon. All of the natural isolates formed well-defined plaques at both temperatures, and there was no apparent difference in the number of plaques formed at these two temperatures. Intrinsic rate of population growth $(r)$ was calculated as $r=\left[\ln \left(N_{45} / N_{0}\right)\right] / 45$.

Our 45-min assay was designed to provide a direct measure of intrinsic growth rate, $r$, in conditions in which resources (hosts) are not limiting and was modeled on previous measures of G4 and $\phi$ X174 fitness in liquid cultures (Bull et al. 2000; Holder and Bull 2001; Knies et al. 2006). At its optimal temperatures, G4 achieves at least two complete rounds of infection and lysis in $45 \mathrm{~min}$ but infects only $0.37 \%$ of the available host cells (assuming a burst size of 298 phage/infected cell [Guyader and Burch 2008] and a single doubling of the bacteria during this time). If we used a longer assay, the phage would exhaust (e.g., kill) the available bacteria, and the limited host availability would impose an upper limit on our measurement of phage growth rate, making it impossible to distinguish phage growth rates at temperatures near the optimum. Lysis times are longer at extreme temperatures than at temperatures near the optimum (J. L. Knies and C. L. Burch, personal observation). In this case, ending the assay at 45 min imposed a downward bias on growth rate estimates for some phages at the most extreme temperatures. Because our assay primarily detects free phages, in cases where lysis time exceeded $45 \mathrm{~min}$, phages will have attached to and infected hosts during this time, and our assay will yield a negative growth rate estimate.

Furthermore, at temperatures near the optimum, the use of a 45-min assay ensured that infection and lysis events had become asynchronous by the end of the assay, so that we were unlikely to measure phage number just before or just after a synchronous lysis event. Longer lysis times at extreme temperatures may have led to increased synchrony and, consequently, increased variance in our estimates of growth rate at these temperatures. 


\section{Characterizing Reaction Norm Shape Variation}

The intrinsic growth rates of the 15 phages were measured three times at each of nine temperatures evenly spaced between $17^{\circ}$ and $41^{\circ} \mathrm{C}$. Growth rate measures were collected in blocks, and individual blocks consisted of a single measurement of all 15 genotypes at one temperature. To remove block effects from our final estimates of continuous reaction norm shapes, we used ANOVA (Sokal and Rohlf 1995) to assess the effects of temperature, genotype, and block (nested within temperature) on $r$ for the natural phage isolates. Genotype and block were treated as class variables, and temperature was treated as a quantitative variable. All the variables were treated as fixed effects. We conducted the analysis using the MIXED procedure in SAS (ver. 8; SAS Institute, Cary, NC), modeling the effect of temperature on $r$ as a third-order polynomial. Residuals were normally distributed (Shapiro-Wilk test: $W=0.98$, $p=.30)$ and homogeneous across temperatures. The resulting estimate for each block effect was subtracted from the growth rate measures from that experiment to produce block-corrected growth rate measures. Corrected growth rate measures were used for all subsequent analyses.

After correcting the growth rate measures for block effects, we estimated continuous reaction norms that describe the relationship between $r$ and temperature for each phage genotype using a statistical method called template mode of variation (TMV; Izem and Kingsolver 2005). We have previously used TMV to analyze thermal reaction norms of phage (Knies et al. 2006). This method assumes that the thermal reaction norms for all phages in the population have a common template shape represented by continuous function $P(t)$. Here the template $P(t)$ is modeled as a third-order polynomial, with positive maximum at $P(0)$ and with $P(t)=0$ for at least two values of $t$ in the neighborhood of $P(0)$. Then the growth rate for each genotype $i$ is given by

$$
P_{i}(t)=a_{i}+\frac{1}{b_{i}} P\left[\frac{1}{b_{i}}\left(t-m_{i}\right)\right] .
$$

The shape of a particular genotype's reaction norm thus changes as a result of differences in the three shape parameters: $a_{i}, m_{i}$, and $b_{i}$, which respectively represent shifts in the average trait height (here, growth rate), optimum temperature, and niche width of a particular reaction norm. The model is fitted by simultaneously optimizing the polynomial coefficients of the template and the parameters $a_{i}$ (height), $m_{i}$ (location of the optimum), and $b_{i}$ (width) for each curve to minimize the sum of squared errors. More details on this method can be found in the study by Izem and Kingsolver (2005).

We examined the common template shape assumption of TMV by comparing the reaction norms estimated by TMV to reaction norms estimated by cubic spline interpolation. Cubic spline interpolation relaxes the assumption of a common shape when fitting a curve to the data. How closely the curve follows the raw data depends on the assigned smoothing parameter. Splines were fitted to each genotype's replicate growth rate measures using the csaps function in MatLab (ver. 6.5; Mathworks). The global smoothing parameter was assigned by fitting splines of different smoothness to the mean growth rate data over all genotypes, and the best-fitting smoothing parameter was quantitatively chosen using leave-one-out cross-validation (Wahba and Wold 1975). For each considered smoothing parameter, this method calculates the sums of squares when each data point is excluded from the spline fit, and the smoothing parameter that minimizes the average of these sums of squares is chosen as the best fit for the data. The smoothing parameters considered here were $0.05,0.1,0.2,0.4,0.8$, and 1.6. The global smoothing parameter of 0.20 minimized the cross-validation error.

Finally, we used a novel application of principal components analysis (PCA) to determine whether variation in reaction norm shape between genotypes is necessarily described by all three of the curve parameters estimated by TMV or whether shape variation between genotypes can be captured in fewer dimensions. The input variables for the PCA were the TMV parameters $a, b$, and $m$ for each of the 15 genotypes; the parameters were standardized by their respective standard deviations because the variables are on different scales of measurement. The output from the PCA was the principal component coefficients or loadings for the three parameters $(a, b$, and $m)$ on the first three principal components and the principal component scores, or the representation of the parameters of each genotype in the principal component space.

To illustrate the reaction norm shapes that lie along the first principal component axis, we used the loadings on PC1 and the scores to calculate new values of $a, b$, and $m$ for each of the 15 genotypes. For a genotype $i$, its new value of $a_{i}$ would be calculated as

$$
a_{i}=\bar{a}+\operatorname{score}_{i, a} \times \text { loading }_{a, 1} \text {, }
$$

where $\bar{a}$ is the mean $a$ across genotypes, score $_{i, a}$ is the score for genotype $i$ on parameter $a$, and loading $a_{a, 1}$ is the loading of $a$ on PC1. This process is repeated for each of the three parameters for all the genotypes. The new $a_{i}, m_{i}$, and $b_{i}$ parameters are then input into equation (1) to generate the reaction norms for each genotype as predicted by PC1. The PCA analysis was conducted using the princomp function in MatLab. 

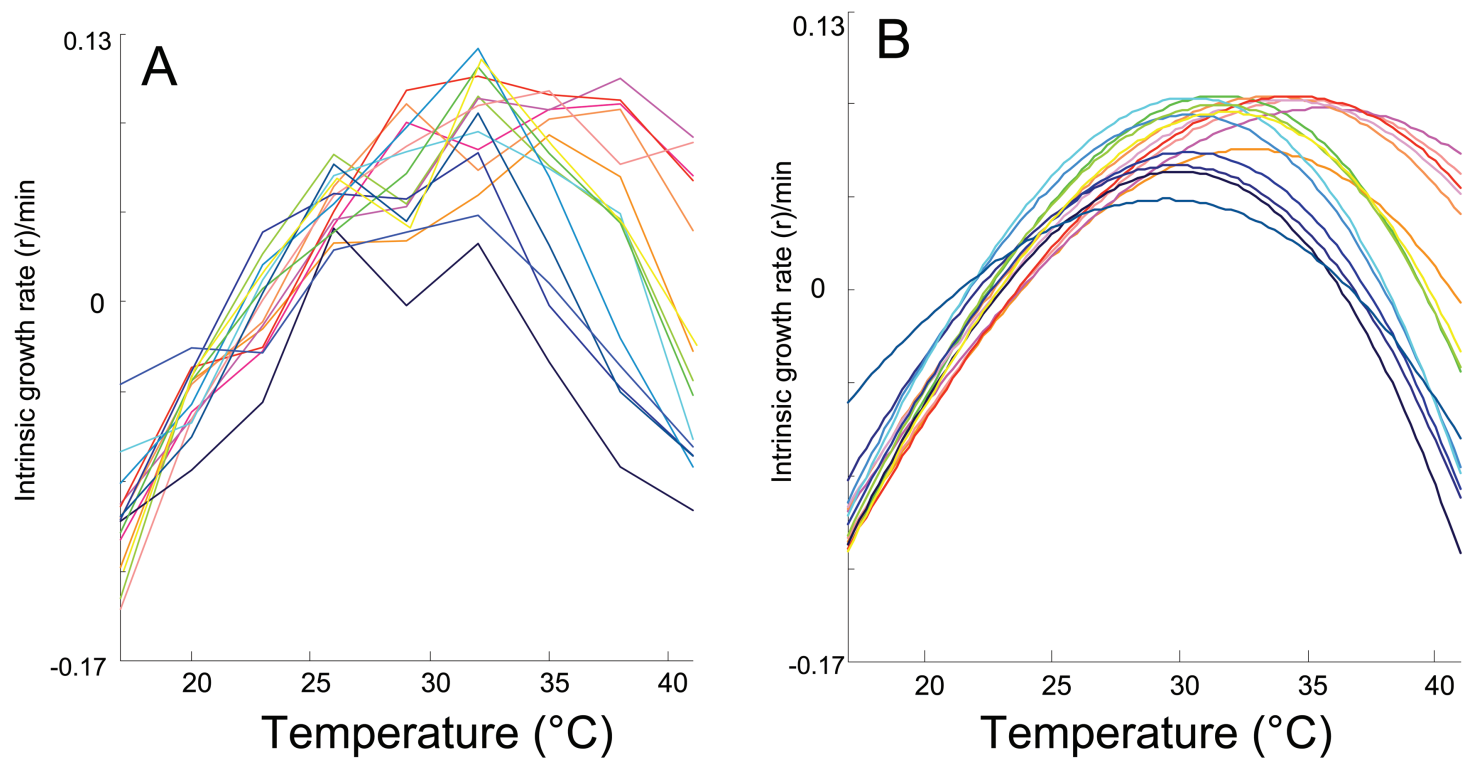

Figure 2: Thermal reaction norms of G4-like phages isolated in nature. The curves are colored according to the $T_{\text {opt }}$ of the phage. The transition is from cold adapted (blue) to hot adapted $(r e d)$. A, Shown are the means of the growth rate measures for each genotype. Each line is a different genotype. $B$, Shown are the continuous reaction norms fitted to the raw data by template mode of variation. The curves were constrained to be a third-order polynomial.

\section{Assessing Correlations between Reaction Norm Characteristics}

The temperature range and $T_{\mathrm{opt}}$ of each genotype were calculated from its TMV-fitted curve. Temperature range was defined as the temperatures for which the genotype has an intrinsic growth rate $\geq 0$. The upper and lower limits of each genotype's temperature range were determined by calculating the $x$-intercepts for each fitted curve. For five of the genotypes, their fitted curves had upper $x$-intercepts greater than the highest experimental temperature $\left(41^{\circ} \mathrm{C}\right)$, and so calculating the $x$-intercept for those curves requires extending the curve over temperatures for which we do not have experimental data. For these genotypes, we assumed that their fitted curves correctly describe their growth rate above $41^{\circ} \mathrm{C}$. We also calculated temperature range for a truncated range that ended at $41^{\circ} \mathrm{C}$.

Pearson's correlation coefficient was used to calculate the correlation between pairs of reaction norm traits (e.g., $T_{\text {opt }}$ and $\left.r_{\text {max }}\right)$. Both simple linear regression and reduced major axis regression were performed on pairs of reaction norm traits. Since the results of both analyses were nearly identical in all cases (the estimates of slope were $<7 \%$ difference), only the simple linear regression results are reported here.

Correlations were calculated for both the uncorrected and the phylogenetically corrected data. For phylogenetic correction, we computed standardized independent contrasts (Felsenstein 1985) using Phenotypic Diversity Analysis Programs (ver. 6.0; Midford et al. 2005; Maddison and Maddison 2006). Phage sequences were downloaded from PubMed (accession numbers reported in Rokyta et al. 2006), and a phylogeny was obtained with MrBayes (ver. 3.1.2; Huelsenbeck and Ronquist 2001) using the GTR + gamma + invariant model of nucleotide substitution and default parameters. Convergence was achieved in 100,000 generations with a $10 \%$ burn-in (10\% of the samples trees was discarded).

\section{Results}

\section{Variation in Thermal Adaptation}

The mean intrinsic growth rate $r$ for phage genotypes increased with temperature until a maximum was achieved around $32^{\circ} \mathrm{C}$ and declined as temperature increased above this maximum (fig. 2A). Substantial variation in $r$ exists between the natural phage isolates, especially at high temperatures. Measures of $r$ were corrected for block effects (ANOVA: temperature $\times$ block, $\mathrm{df}=25$, Type III $F=$ $8.93, p<.0001)$, and continuous reaction norms were estimated from the corrected data using the statistical 
method TMV (fig. 2B). The TMV method attempts to improve the precision of reaction norm estimates by assuming the existence of a common curve shape. We confirmed that this assumption was reasonable by additionally fitting the data with a curve using cubic spline interpolation (a method that does not assume a common shape). The curves fit by the two methods were very similar (fig. 3).

\section{General Shape of Thermal Reaction Norms}

To determine the extent to which the parameters that describe reaction norm shape covary, we performed a PCA on the data set containing the parameters $a, b$, and $m$ for all 15 phages. The first principal component explains a large amount $(72.24 \%)$ of the variation in these parameters, while the second and third PCs explain an additional $24.9 \%$ and $2.86 \%$. PC1 loadings were similar in magnitude for all three parameters, positively for $a$ and $m$ and negatively for $b$ (fig. $4 A$ ). Since PC1 explains so much variation in these parameters, it appears that variation in reaction norm shape is constrained. To visualize this constraint on reaction norm shape, the loadings of each parameter on $\mathrm{PC} 1$, along with the PC1 scores for each genotype, were used to calculate the PC1 parameter values for each genotype. These new parameter values were input into the TMV equation (eq. [1]) and used to generate the PC1 expected reaction norm shape for each genotype (fig. $4 B$; the PC2 expectations are also shown in fig. 4C). This major component of reaction norm shape variation is consistent with hotter is better because $r$ at $T_{\text {opt }}\left(r_{\text {max }}\right)$ increases with $T_{\text {opt }}$ (see fig. 1).

\section{Hotter Is Better}

The optimal temperature $\left(T_{\text {opt }}\right)$, the intrinsic growth rate at the optimal temperature (i.e., maximum intrinsic growth rate; $r_{\max }$ ), and the temperature range (defined as the temperature range over which intrinsic growth rate is positive) were calculated directly for each genotype from the continuous reaction norms estimated by TMV. $T_{\text {opt }}$ estimates varied between $28.7^{\circ}$ and $35.5^{\circ} \mathrm{C}, r_{\max }$ estimates
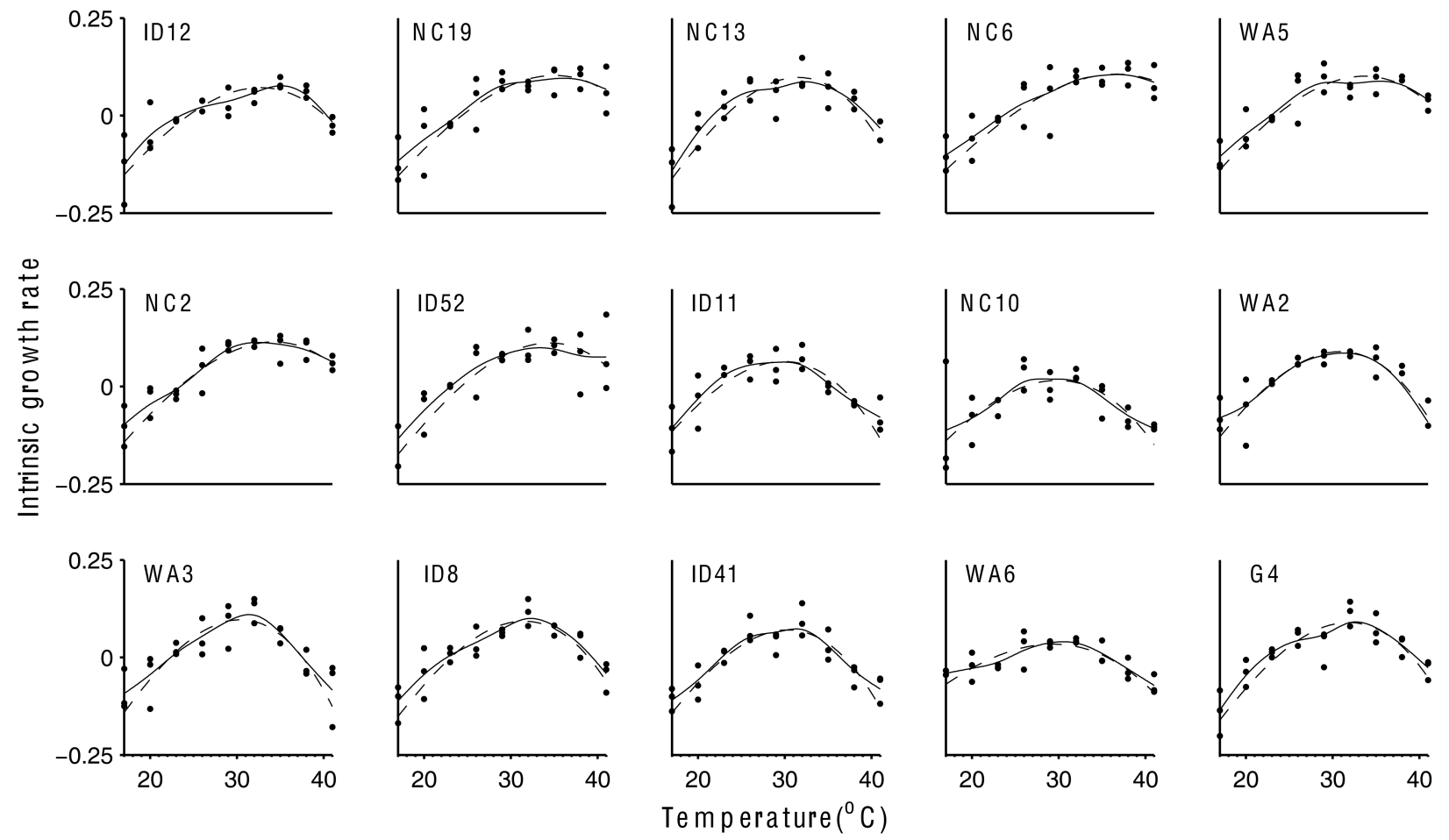

Figure 3: Curves are fitted to the growth rate data for each genotype using smoothing splines (solid lines) and template mode of variation (TMV; dashed lines). TMV assumes that all the curves share a common shape (here a third-order polynomial). The smoothing splines do not assume a common shape. The TMV assumption of a common shape of the reaction norms is validated by the close match of the curves fitted by TMV and the smoothing splines to each data set. The black circles in each panel are the three replicate growth rate measures at each temperature. The genotype name is in the upper left corner of each panel. 

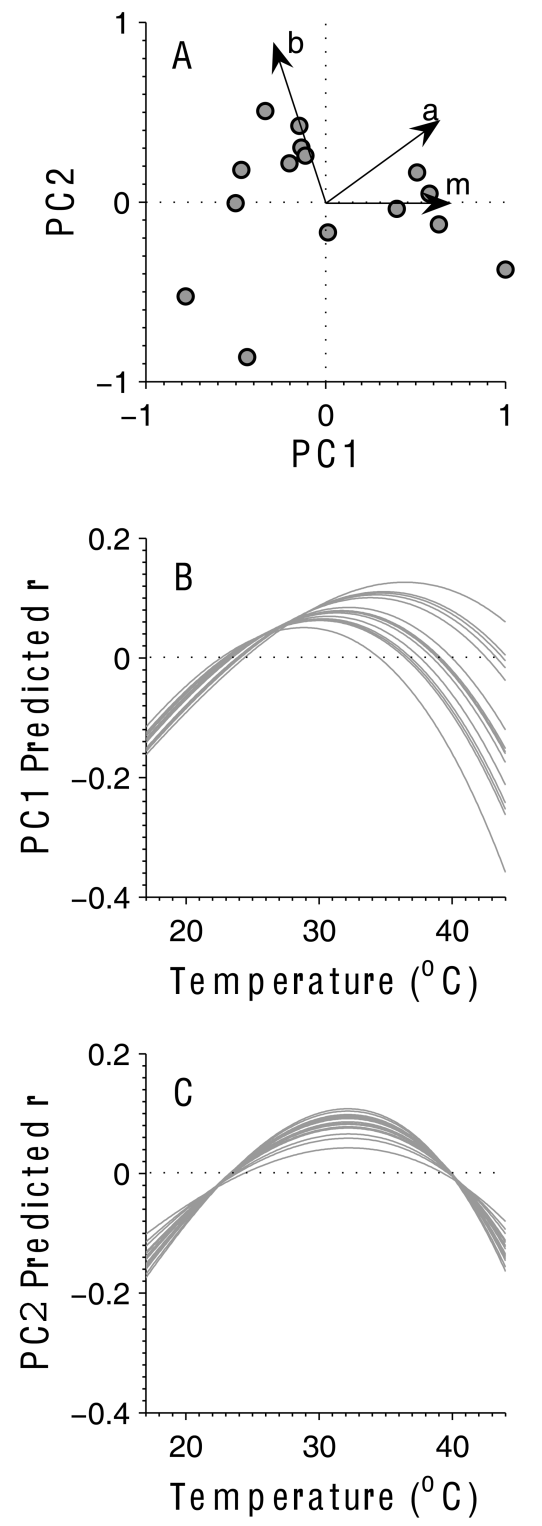

Figure 4: Principal components analysis of phage thermal reaction norms. A, The loadings of the parameters (arrows) and the scores (circles) for the natural phage isolates on PC1 and PC2. B, For each genotype, the predicted reaction norms were plotted using the parameter values $(a, b$, and $m)$ defined by PC1. C, Predicted reaction norms plotted using the parameter values defined by PC2. The component loadings of $a, b$, and $m$ on PC1, PC2, and PC3, respectively, were as follows: $a: 0.58,0.56$, $-0.59 ; b$ : $-0.47,0.82,0.32 ; m: 0.66,0.094,0.74$.

varied between 0.044 and 0.11 (8.9-148.4-fold increase in phages per $45 \mathrm{~min}$ ), and temperature range varied between $14.7^{\circ}$ and $22.3^{\circ} \mathrm{C}$. Five of the genotypes had upper temperature limits $\left(44.6^{\circ}, 46.1^{\circ}, 43.1^{\circ}, 43.7^{\circ}\right.$, and $\left.43.6^{\circ} \mathrm{C}\right)$ greater than the highest experimental temperature $\left(41^{\circ} \mathrm{C}\right)$. If we assign the upper temperature limit of these five ge- notypes as $41^{\circ} \mathrm{C}$, then temperature range over all genotypes varied between $14.7^{\circ}$ and $18.2^{\circ} \mathrm{C}$.

There were significant positive correlations between $T_{\mathrm{opt}}$ and $r_{\max }$ (Pearson's $r=0.91, \mathrm{df}=13, p<.0001$; fig. 5A) and between $T_{\mathrm{opt}}$ and temperature range (Pearson's $r=$ 0.98 , df $=13, p<.0001$; fig. $5 B$ ). Both correlations remained significant and positive after correcting for the relatedness of the phages using Felsenstein's phylogenetic independent contrasts (PIC; $T_{\text {opt }}$ and $r_{\max }$ : Pearson's $r=$ $0.94, \mathrm{df}=13, p<.0001 ; T_{\text {opt }}$ and temperature range: Pearson's $r=0.94$, $\mathrm{df}=13, p<.0001)$. The correlation between $T_{\text {opt }}$ and temperature range decreased slightly to $r=0.88$ when the upper temperature limit was truncated at $41^{\circ} \mathrm{C}$ but remained highly significant $(\mathrm{df}=13, p<$ .0001). There was no significant association between geographic location and $T_{\text {opt }}$ (group 1: ID and WA phages; group 2: NC phages; $t=2.04, \mathrm{df}=12, p=.06)$ or between geographic location and temperature range (group one: ID and WA phages; group 2: NC phages; $t=1.28$, $\mathrm{df}=12, p=.11$ ).

The quantitative dependence of $r_{\max }$ on $T_{\mathrm{opt}}$ should follow the exponential temperature dependence model, specifically, $\ln r \propto-E / k T$, where $E$ is the activation energy of the rate limiting metabolic reaction and $k$ is the Boltzmann constant (Gillooly et al. 2001). $E$ is then estimated as the slope of the regression of $\ln r$ on $1 / k T$. For the G4-like natural isolates, there was a linear relationship between $\ln r_{\max }$ and $1 / k T_{\text {opt }}$ indicating that the phages are unable to compensate for the rate-depressing effects of low temperature. The estimated slope was $-0.85 \mathrm{eV}(E=0.85$ eV; 95\% confidence interval [CI]: 0.57-1.13; PIC: $E=$ $1.08 \mathrm{eV} ; 95 \%$ CI: $0.82-1.33$ ) for the phage data set (fig. $5 C$, dashed line).

Since the phages use their host's proteins to replicate their genome (Dumas et al. 1979), the sensitivity of the phages' growth rate to temperature may depend on the thermal sensitivity of their host, Escherichia coli. To investigate this possibility, we reanalyzed the data of Bronikowski et al. (2001) to estimate the temperature dependence of E. coli. This data set consisted of growth rate measures at temperatures between $10^{\circ}$ and $45^{\circ} \mathrm{C}$ for 143 E. coli individuals isolated from three different natural populations over 2 years. Continuous reaction norms were fit to the data using TMV, and $r_{\max }$ and $T_{\text {opt }}$ were calculated from the resulting curves. For these E. coli, the slope of $\ln r_{\max }$ on $1 / k T_{\text {opt }}$ is $-0.38(E=0.38 \mathrm{eV} ; 95 \%$ CI: $0.33-$ 0.42 ; fig. $5 D$, solid line). For comparative purposes, a regression line with a slope equal to the phage estimate is shown through the mean E. coli data (cf. dashed and solid lines in fig. 5D). It appears that the G4-like phages are more sensitive to changes in temperature than their host. 

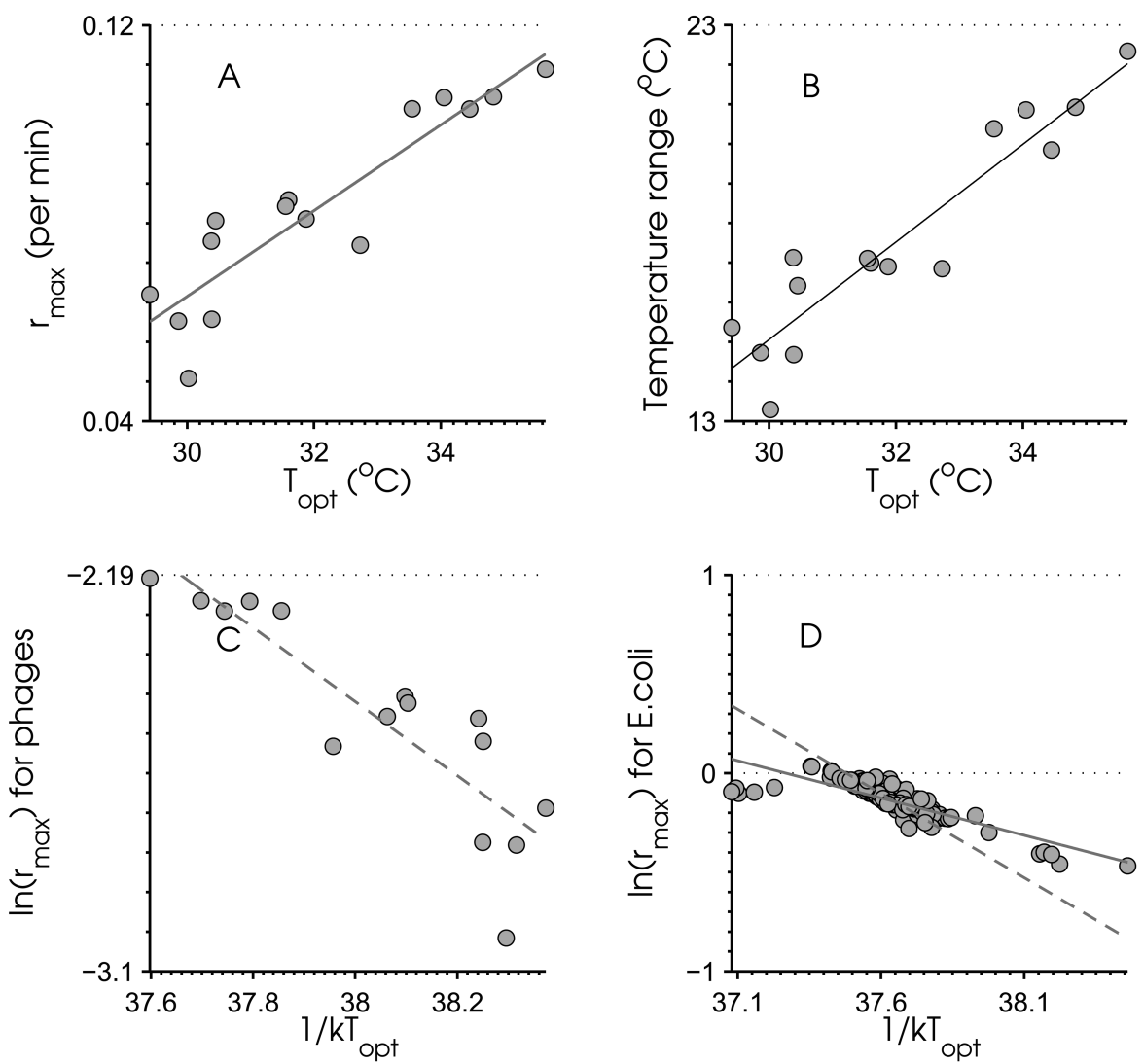

Figure 5: Correlation between reaction norm traits for the phages and for Escherichia coli. $A-C$ are data for the natural isolates of G4, and $D$ is data for natural isolates of $E$. coli. A, As predicted by hotter is better, a positive correlation exists between $r_{\max }$ and $T_{\text {opt }}$. B, A positive correlation also exists between temperature range and $T_{\mathrm{opt}}$. C, For the phages, a negative correlation exists between $1 / k T_{\mathrm{opt}}$ and $\ln r_{\max }$. D, For E. coli, a negative correlation also exists between $1 / k T_{\text {opt }}$ and $\ln r_{\max }$. For comparative purposes, the dashed line shows the expected regression line if $E$. coli responded to changes in temperature like G4.

\section{Discussion}

\section{Hotter Is Better}

In this article, we measured thermal reaction norms for intrinsic growth rate $(r)$ in a collection of 15 G4-like phages to test the hotter is better hypothesis. This hypothesis predicts that adaptation will be unable to overcome the thermodynamic constraints that limit rates of enzyme activity at cold temperatures and that, as a result, genotypes with higher $T_{\text {opt }}$ will also exhibit higher $r_{\max }\left(r\right.$ at $T_{\text {opt }}$; as in Frazier et al. 2006). Our data confirmed this prediction. The $T_{\text {opt }}$ and $r_{\max }$ of the 15 phages were positively correlated, and the strength of the correlation (Pearson's $r=$ 0.91; PIC: Pearson's $r=0.94)$ suggests an absolute constraint in the way that thermal reaction norms can vary. In our data set, for every $1^{\circ} \mathrm{C}$ decrease in optimal temperature, $r_{\max }$ of the natural phage decreases by 0.0089 (PIC: 0.011$)$. For the phages with the highest $T_{\text {opt }}$ 's, this value $(0.0089)$ is an $8 \%$ decrease in $r_{\max }$.
Our experiment is the first intraspecific study to document a significant positive correlation between fitness $\left(r_{\max }\right)$ and $T_{\text {opt }}$. This finding complements the comparative analyses of Frazier et al. (2006) that demonstrated a strong positive correlation between $r_{\max }$ and $T_{\text {opt }}$ across insect species. Though it is possible that hotter is better may not apply to all performance traits (e.g., Herron 1996; Izem and Kingsolver 2005), the current evidence strongly supports the hotter is better hypothesis for overall fitness.

Our study also stands out from most previous studies of the thermal sensitivity of fitness because we measured the intrinsic rate of population growth $(r)$ directly, using a standard assay for measuring phage fitness in liquid (Bull et al. 2000; Holder and Bull 2001). In contrast, most previous studies measured age-specific survival and fecundity schedules for a cohort of individuals and then used Euler's equation to estimate $r$ (Roff 2002). This approach to estimating fitness requires several assumptions that our approach did not, including the assumptions that survival 
and fecundity schedules are constant and that the population has achieved a stable age distribution and constant exponential growth (Roff 2002).

\section{Underlying Causes of Hotter Is Better}

Although the positive correlation that we observed between $T_{\text {opt }}$ and $r_{\max }$ was predicted from a consideration of known thermodynamic effects of temperature on reaction rates, other mechanistic and evolutionary explanations are possible. An alternative mechanistic explanation is that the slower growth rate of the host bacterium (Escherichia coli strain C) at the colder temperatures examined in this study may have limited phage growth rates at these temperatures. This alternative explanation for the hotter is better pattern seems unlikely because the growth rates of the phage are more sensitive to temperature than the growth rates of their host, E. coli.

Evolutionary explanations are also possible. In particular, the hotter is better pattern would emerge if lower $r_{\max }$ evolved as a correlated response to selection for broader temperature ranges in low-temperature environments (Levins 1968; Huey and Slatkin 1976; Gilchrist 1995; Portner 2004; Frazier et al. 2006). If this alternative hypothesis explained our data, we would expect to see a trade-off between the temperature range and the $r_{\max }$ of the phages. Our data reveal the opposite pattern: temperature range and $r_{\max }$ are strongly positively correlated. Phage with low $T_{\text {opt }}$ exhibited both narrow temperature ranges and low $r_{\max }$, whereas phage with high $T_{\text {opt }}$ exhibited both wide temperature ranges and high $r_{\max }$. Thus, our data allow us to reject this evolutionary explanation for the hotter is better pattern.

\section{The Thermal Sensitivity of Fitness}

The model of Gillooly et al. (2001) and Savage et al. (2004) predicts how metabolic reaction rates and fitness $(r)$ depend on temperature and size, on the basis of the predicted effects of temperature $(T)$ and size $(M)$ on metabolic reaction rates $(B)$. Formally, $B(M, T) \propto M^{3 / 4} e^{-E / k T}$. Replacing metabolic reaction rates with intrinsic rate of population growth (Savage et al. 2004) and normalizing this rate by size, this equation is written as $r / M^{3 / 4} \propto e^{-E / k T}$. Since the phages are invariant in size, this equation can be rewritten without the size term as $r \propto e^{-E / k T}$. This model predicts a linear relationship between $\ln r$ and 1/kT. The slope of this relationship, $E$, is thus a measure of thermal sensitivity of $r$. Gillooly et al. (2001) named this slope $E$, the activation energy of metabolic-limiting reactions. If $E$ is constant across temperatures and genotypes, then we predict that a linear relationship will exist between measures of $\ln r_{\max }$ and $1 / k T_{\mathrm{opt}}$, so that estimates of $E$ represent the quantitative strengths of the hotter is better effect. Consistent with our prediction, there is a linear relationship between $\ln r_{\max }$ and $1 / k T_{\text {opt }}$. The slope of this relationship, $E$, is $0.85 \mathrm{eV}$ (95\% CI: $0.57-1.13$; PIC: $E=1.08 \mathrm{eV}$; $95 \%$ CI: $0.82-1.33)$. Our estimate of the thermal sensitivity of phage $E$ is not statistically distinguishable from the estimate obtained by Frazier et al. (2006) in a comparative analysis across insect species $(E=0.97$; $95 \%$ CI: $0.78-$ 1.20). However, our and Frazier et al.'s (2006) estimates of the thermal sensitivity of fitness are greater than those of Savage et al. (2004; average of $0.6 \mathrm{eV}$ ). The estimate of $E$ from Savage et al. (2004) comes from investigations of the relationship between $\ln r$ and $1 / k T$ across higher taxonomic orderings. This difference in the thermal sensitivity of fitness, $E$, between our study and that of and Savage et al. (2004) requires further investigation.

\section{Hotter Is Broader}

Although we started with an a priori expectation that phage reaction norms would show the hotter is better pattern, we were surprised to find that hotter is also broader. Previous models of reaction norm evolution assume a constant area under the curve (Levins 1968; Gilchrist 1995). If hotter is better, then the constant area under the curve will be maintained as a decrease in temperature range. Although increases in $T_{\mathrm{opt}}$ are accompanied by slight decreases in growth rate at the lowest temperatures (figs. $2 B, 4 B$ ), for every $1^{\circ} \mathrm{C}$ increase in optimal temperature, the temperature range increased by $1.23^{\circ} \mathrm{C}$. As a result, the total positive area under phage reaction norms increases dramatically with increasing $T_{\mathrm{opt}}$.

We considered whether this correlation between $T_{\text {opt }}$ and temperature range could be explained by the manner in which these phages were isolated from nature, our measure of intrinsic growth rate $(r)$, or by chance preadaptation of certain genotypes to the lab culture conditions. The initial isolation of these phages from nature required that the phages survive and form visible plaques at $37^{\circ} \mathrm{C}$ (Rokyta et al. 2006). This screen at $37^{\circ} \mathrm{C}$ would be expected to disproportionally eliminate phage with low optimal temperatures and poor growth at $37^{\circ} \mathrm{C}$ (i.e., phage with a low $T_{\text {opt }}$ and a narrow temperature range). If anything, the phage isolation method would be expected to bias against the observed positive correlation between $T_{\mathrm{opt}}$ and temperature range. The method we used to measure $r$ was designed to maximize accuracy at temperatures near the optimum. At extreme temperatures, we are more likely to terminate the assay just before or after the phage lyse the host cells, and as a result, measurement error may increase as $r$ approaches and falls below zero. However, this measurement error will increase at both high and low temperatures and is not expected to impose a systematic bias 


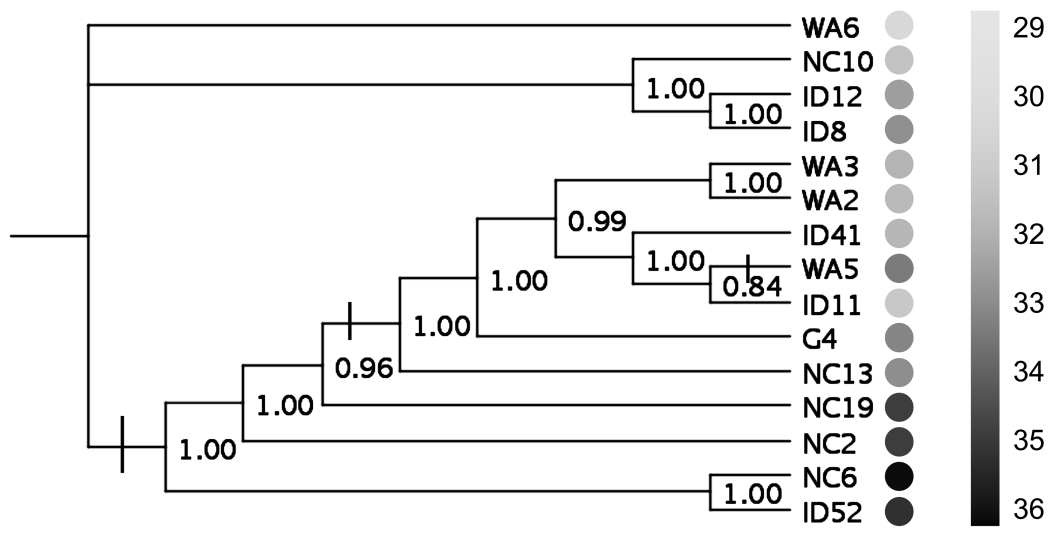

Figure 6: Phylogeny of the natural isolates of the G4-like phage with the estimated switches between hot and cold optimal temperatures. Solid black bars indicate the estimated switches. Each genotype is shown in its position on the phylogeny along with its optimal temperature. The scaling for $T_{\text {opt }}$ is shown on the right. The probability of each partition is indicated by the number at each node. A color version of this figure is available in the online edition of the American Naturalist.

that affects our conclusions. Finally, we find it difficult to rule out the possibility that the hotter is broader pattern is explained by chance differences in preadaptation to the lab conditions (Service and Rose 1985) among these phages. However, this explanation would require that preadaptation to the lab conditions improved fitness more at high temperatures than at low, and we do not have data to suggest this relationship. Moreover, in a previous experiment conducted using a G4 genotype that was preadapted to the lab, we observed that phage adaptation to high temperature (in the lab) resulted in phages with both higher optimal temperatures and broader thermal ranges (Knies et al. 2006). These data confirm the existence of genetic variation capable of producing the hotter is broader pattern in these phages.

Experiments in E. coli also confirm the existence of genetic variation capable of producing the hotter is broader pattern but suggest that this pattern may be characteristic only of populations that experience a variable thermal environment. For example, Leroi et al. (1994) adapted lines of $E$. coli to alternating temperatures at $32^{\circ}$ and $42^{\circ} \mathrm{C}$. The resulting lines had increased fitness at both $32^{\circ}$ and $42^{\circ} \mathrm{C}$, relative to ancestral lines; importantly, the mean fitness increase at $42^{\circ} \mathrm{C}$ was much greater $(20 \%)$ than at $32^{\circ} \mathrm{C}$ (4\%). In contrast, long-term laboratory evolution at constant temperatures $\left(32^{\circ}, 37^{\circ}\right.$, or $\left.42^{\circ} \mathrm{C}\right)$ resulted in no detectable changes in the temperature range of experimental lines of E. coli (Bennett and Lenski 1993). These results suggest that under variable thermal conditions, adaptation can increase thermal breadth (e.g., the area under the fitness curve across these temperatures) and increase performance at higher temperatures. The phages in our study most likely experienced selection at a range of temperatures in their natural environment.

The positive correlation between $T_{\mathrm{opt}}$ and temperature range we observed could result from two different historical scenarios: adaptation to high temperatures correlated with an extended temperature range or adaptation to low temperatures correlated with a reduced temperature range. We can use data on phage relatedness to map $T_{\mathrm{opt}}$ onto the phylogeny of the phage (fig. 6). This mapping suggests that the ancestral condition of the phage was a low $T_{\text {opt }}$ (WA6 clade: $T_{\text {opt }}$ varies between $29.41^{\circ}$ and $32.73^{\circ} \mathrm{C}$ ) and that a high $T_{\text {opt }}$ evolved between the split between the WA6 clade and all the other genotypes. A second transition to a low $T_{\text {opt }}$ then occurred after the split between genotypes NC19 and NC13. This admittedly crude analysis tentatively supports both hypotheses: adaptation to a high $T_{\text {opt }}$ is positively correlated with temperature range, and adaptation to a low $T_{\text {opt }}$ is negatively correlated with temperature range.

The positive correlation between adaptation to a high $T_{\text {opt }}$ and increased temperature range is puzzling because generalist-specialist trade-offs have been observed at the biochemical level between enzyme stability and performance (Fields 2001) and are expected to produce generalist-specialist trade-offs at the level of fitness (Angilletta 2006). While the literature on generalist-specialist tradeoffs says nothing about $T_{\mathrm{op} \text {, }}$ the presence of such generalist-specialist trade-offs should result in a narrowing of temperature range for phage with a high $T_{\text {opt }}$ and high $r_{\max }$. Our results suggest that either these trade-offs do exist and are compensated for at higher physiological levels in this phage population or these trade-offs are not universal. 
In sum, our results indicate that adaptation to high temperatures is a win-win situation. Not only is adaptation to high temperatures less constrained by the thermodynamic limits on population growth at low temperatures, but also adaptation to high temperatures pays very little cost for growth at low temperature, achieving both higher maximum growth rates and a broader temperature range.

\section{Acknowledgments}

We thank S. Marron and J. Weiss for their conversations from which we were able to improve our analysis of the phage growth rate data and the cubic spline interpolation to the data. H. Oloffson was often helpful in MatLab debugging. A. Bronikowski graciously sent us the raw Escherichia coli data. We thank M. Angilletta, R. A. Enke, D. Weinreich, members of the Burch lab, and an anonymous reviewer for comments that improved the analysis and final manuscript. This work was supported in part by National Science Foundation (NSF) EF-0328594 (to J.G.K.), National Institutes of Health GM067940 (to C.L.B.), and NSF 0710044 (to J.L.K.).

\section{Literature Cited}

Angilletta, M. J. 2006. Estimating and comparing thermal performance curves. Journal of Thermal Biology 31:541-545.

Angilletta, M. J., P. H. Niewiarowski, and C. A. Navas. 2002. The evolution of thermal physiology in ectotherms. Journal of Thermal Biology 27:249-268.

Bauwens, D., T. Garland, A. M. Castilla, and R. Vandamme. 1995. Evolution of sprint speed in lacertid lizards: morphological, physiological, and behavioral covariation. Evolution 49:848-863.

Bennett, A. 1987. Evolution of the control of body temperature: is warmer better? Pages 421-431 in P. Dejours, L. Bolis, C. Taylor, and E. Weibel, eds. Comparative physiology: life in water and on land. Liviana, Padova.

Bennett, A. F., and R. E. Lenski. 1993. Evolutionary adaptation to temperature. 2. Thermal niches of experimental lines of Escherichia coli. Evolution 47:1-12.

Bowler, K., and A. R. Cossins. 1987. Temperature biology of animals. Chapman \& Hall, New York.

Bronikowski, A. M., A. F. Bennett, and R. E. Lenski. 2001. Evolutionary adaptation to temperature. VII. Effects of temperature on growth rate in natural isolates of Escherichia coli and Salmonella enterica from different thermal environments. Evolution 55:3340.

Bull, J. J., M. R. Badgett, and H. A. Wichman. 2000. Big-benefit mutations in a bacteriophage inhibited with heat. Molecular Biology and Evolution 17:942-950.

Dumas, L. B., S. Goltz, and D. T. Benesh. 1979. Host cell DNA initiation protein requirements for replication of bacteriophage G4 replicative-form DNA. Journal of Virology 31:370-375.

Eppley, R. W. 1972. Temperature and phytoplankton growth in the sea. Fishery Bulletin 70:1063-1085.

Felsenstein, J. 1985. Phylogenies and the comparative method. American Naturalist 125:1-15.

Fiddes, J. C., B. G. Barrell, and G. N. Godson. 1978. Nucleotide sequences of the separate origins of synthesis of bacteriophage G4 viral and complementary DNA strands. Proceedings of the Royal Society B: Biological Sciences 75:1081-1085.

Fields, P. A. 2001. Review: protein function at thermal extremes: balancing stability and flexibility. Comparative Biochemistry and Physiology A 129:417-431.

Frazier, M. R., R. B. Huey, and D. Berrigan. 2006. Thermodynamics constrains the evolution of insect population growth rates: "warmer is better." American Naturalist 168:512-520.

Gilchrist, G. W. 1995. Specialists and generalists in changing environments. 1. Fitness landscapes of thermal sensitivity. American Naturalist 146:252-270.

Gillooly, J. F., J. H. Brown, G. B. West, V. M. Savage, and E. L. Charnov. 2001. Effects of size and temperature on metabolic rate. Science 293:2248-2251.

Guyader, S., and C. L. Burch. 2008. Optimal foraging predicts the ecology but not the evolution of host specialization in bacteriophages. PLoS ONE 3:e1946.

Hamilton, W. 1973. Life's color code. McGraw-Hill, New York.

Heilmayer, O., T. Brey, and H. O. Portner. 2004. Growth efficiency and temperature in scallops: a comparative analysis of species adapted to different temperatures. Functional Ecology 18:641-647.

Herron, J. 1996. Genetic variation, thermal sensitivity, and thermal acclimation in Volvox aureus and Volvox globator. PhD diss. University of Washington, Seattle.

Hochachka, P., and G. Somero. 2002. Biochemical adaptation: mechanism and process in physiological evolution. Academic Press, New York.

Holder, K. K., and J. J. Bull. 2001. Profiles of adaptation in two similar viruses. Genetics 159:1393-1404.

Huelsenbeck, J., and F. Ronquist. 2001. MrBayes: Bayesian inference of phylogenetic trees. Bioinformatics 17:754-755.

Huey, R. B., and P. Hertz. 1984. Is a jack-of-all-temperatures a master of none? Evolution 38:441-444.

Huey, R. B., and J. G. Kingsolver. 1989. Evolution of thermal sensitivity of ectotherm performance. Trends in Ecology \& Evolution 4:131-135.

Huey, R. B., and M. Slatkin. 1976. Cost and benefits of lizard thermoregulation. Quarterly Review of Biology 51:363-384.

Izem, R., and J. G. Kingsolver. 2005. Variation in continuous reaction norms: quantifying directions of biological interest. American Naturalist 166:277-289.

Johnston, I. A., and A. F. Bennett. 1996. Animals and temperature: phenotypic and evolutionary adaptation. Cambridge University Press, New York.

Knies, J. L., R. Izem, K. L. Supler, J. G. Kingsolver, and C. L. Burch. 2006. The genetic basis of thermal reaction norm evolution in lab and natural phage populations. PloS Biology 4:1257-1264.

Leroi, A. M., R. E. Lenski, and A. F. Bennett. 1994. Adaptation of Escherichia coli to a temporally varying environment. Evolution 48:1222-1229.

Levins, R. 1968. Evolution in changing environments: some theoretical explorations. Princeton University Press, Princeton, NJ.

Lynch, M., and W. Gabriel. 1987. Environmental tolerance. American Naturalist 129:283-303.

Maddison, W. P., and D. R. Maddison. 2006. Mesquite: a modular system for evolutionary analysis. Version 1.1. http:// mesquiteproject.org.

Midford, P. E., T. Garland Jr., and W. P. Maddison. 2005. PDAP 
package of Mesquite. Version 1.07. http://mesquiteproject.org/ pdap_mesquite/index.html.

Palys, T., L. K. Nakamura, and F. M. Cohan. 1997. Discovery and classification of ecological diversity in the bacterial world: the role of DNA sequence data. International Journal of Systematic Bacteriology 47:1145-1156.

Portner, H. O. 2004. Climate variability and the energetic pathways of evolution: the origin of endothermy in mammals and birds. Physiological and Biochemical Zoology 77:959-981.

Precht, H., J. Christophersen, H. Hensel, and W. Larcher. 1973. Temperature and life. Springer, Berlin.

Rehfeld, G. E., N. M. Tchebakova, Y. I. Parfenova, W. R. Wykoff, N. A. Kuzmina, and L. I. Milyutin. 2002. Intraspecific responses to climate in Pinus sylvestris. Global Change Biology 8:912-929.

Roff, D. A. 2002. Life history evolution. Sinauer, Sunderland, MA.

Rokyta, D. R., C. L. Burch, S. B. Caudle, and H. A. Wichman. 2006. Horizontal gene transfer and the evolution of microvirid coliphage genomes. Journal of Bacteriology 188:1134-1142.
Savage, V. M., J. F. Gillooly, J. H. Brown, G. B. West, and E. L. Charnov. 2004. Effects of body size and temperature on population growth. American Naturalist 163:429-441.

Service, P. M., and M. R. Rose. 1985. Genetic covariation among lifehistory components: the effect of novel environments. Evolution 39:943-945.

Sokal, R. R., and F. J. Rohlf. 1995. Biometry. W. H. Freeman, New York.

Wahba, G., and S. Wold. 1975. A completely automatic french curve: fitting spline functions by cross validation. Communications in Statistics: Theory and Methods 4:1-17.

Yamahira, K., M. Kawajiri, K. Takeshi, and T. Irie. 2007. Inter- and intrapopulation variation in thermal reaction norms for growth rate: evolution of latitudinal compensation in ectotherms with a genetic constraint. Evolution 61:1577-1589.

Associate Editor: Raymond B. Huey Editor: Monica A. Geber 